\title{
VISITORS FROM THE HALO: 11 Gyr OLD WHITE DWARFS IN THE SOLAR NEIGHBORHOOD*
}

\author{
Mukremin Kilic ${ }^{1,8}$, Jeffrey A. Munn ${ }^{2}$, Kurtis A. Williams ${ }^{3}$, P. M. Kowalski ${ }^{4,5}$, Ted von Hippel ${ }^{6}$, Hugh C. Harris ${ }^{2}$, \\ Elizabeth J. Jeffery ${ }^{7}$, Steven DeGennaro ${ }^{3}$, Warren R. Brown ${ }^{1}$, and B. McLeod ${ }^{1}$ \\ ${ }^{1}$ Smithsonian Astrophysical Observatory, 60 Garden Street, Cambridge, MA 02138, USA; mkilic@ cfa.harvard.edu \\ ${ }^{2}$ US Naval Observatory, P.O. Box 1149, Flagstaff, AZ 86002, USA \\ ${ }^{3}$ Department of Astronomy, 1 University Station C1400, Austin, TX 78712, USA \\ ${ }^{4}$ Helmholtz-Centre Potsdam-GFZ German Research Centre for Geosciences, D-14473 Potsdam, Germany \\ ${ }^{5}$ Lehrstuhl für Theoretische Chemie, Ruhr-Universität Bochum, 44780 Bochum, Germany \\ ${ }^{6}$ Physics Department, Siena College, 515 Loudon Road, Loudonville, NY 12211, USA \\ ${ }^{7}$ Space Telescope Science Institute, 3700 San Martin Drive, Baltimore, MD 21218, USA \\ Received 2010 January 15; accepted 2010 April 6; published 2010 April 28
}

\begin{abstract}
We report the discovery of three nearby old halo white dwarf (WD) candidates in the Sloan Digital Sky Survey (SDSS), including two stars in a common proper motion binary system. These candidates are selected from our $2800 \mathrm{deg}^{2}$ proper motion survey on the Bok and U.S. Naval Observatory Flagstaff Station $1.3 \mathrm{~m}$ telescopes, and they display proper motions of 0 ' $4-0.5 \mathrm{yr}^{-1}$. Follow-up MMT spectroscopy and near-infrared photometry demonstrate that all three objects are hydrogen-dominated atmosphere WDs with $T_{\text {eff }} \approx 3700-4100 \mathrm{~K}$. For average mass WDs, these temperature estimates correspond to cooling ages of 9-10 Gyr, distances of 70-80 pc, and tangential velocities of $140-200 \mathrm{~km} \mathrm{~s}^{-1}$. Based on the $U V W$ space velocities, we conclude that they most likely belong to the halo. Furthermore, the combined main-sequence and WD cooling ages are 10-11 Gyr. Along with SDSS J1102+4113, they are the oldest field WDs currently known. These three stars represent only a small fraction of the halo WD candidates in our proper motion survey, and they demonstrate that deep imaging surveys like the Pan-STARRS and Large Synoptic Survey Telescope should find many old thick disk and halo WDs that can be used to constrain the age of the Galactic thick disk and halo.
\end{abstract}

Key words: stars: atmospheres - stars: evolution - white dwarfs

Online-only material: color figures

\section{INTRODUCTION}

White dwarf (WD) cosmochronology provides an independent and accurate age dating method for different Galactic populations (Winget et al. 1987; Liebert et al. 1988). Using 43 cool WDs in the solar neighborhood, Leggett et al. (1998) derived a disk age of $8 \pm 1.5$ Gyr. Kilic et al. (2006) and Harris et al. (2006) significantly improved the field WD sample by using Sloan Digital Sky Survey (SDSS) and USNO-B astrometry to select high proper motion candidates. However, their survey suffered from the magnitude limit of the Palomar Observatory Sky Survey plates, and they were unable to find many thick disk or halo WD candidates.

Substantial investment of the Hubble Space Telescope time on two globular clusters, M4 and NGC 6397, revealed clean WD cooling sequences. Hansen et al. (2004, 2007) and Bedin et al. (2009) use these data to derive cooling ages of $\approx 12 \mathrm{Gyr}$ for the two clusters. The coolest WDs in these clusters are about 650 $\pm 230 \mathrm{~K}$ cooler than the coolest WDs in the disk (Kowalski 2007). These studies demonstrate that the Galactic halo is older than the disk by $\geqslant 2$ Gyr (Hansen et al. 2002; Fontaine et al. 2001; Kowalski 2007). Even though the WDs in globular clusters provide reliable age estimates, these clusters may not represent the full age range of the Galactic halo. The required exposure times to reach the WD terminus in globular clusters limit these studies to the nearest few clusters. In addition, only two-filter $(V$ and $I)$ photometry is used to model the absolute

\footnotetext{
* Based on observations obtained at the MMT Observatory, a joint facility of the Smithsonian Institution and the University of Arizona.

8 Spitzer Fellow.
}

magnitude and color distribution of the oldest WDs to derive ages. The far closer and brighter WDs of the local halo field are an enticing alternative as well as complementary targets, with the additional potential to constrain the age range of the Galactic halo. Accurate ages for field WDs can be obtained through optical and near-infrared photometry and trigonometric parallax measurements. Nearby WDs can also be used to understand the model uncertainties and put the Globular cluster ages on a more secure footing.

The quest for field halo WDs has been hampered by the lack of proper motion surveys that go deep enough to find the cool halo WDs. The initial claims for a significant population of halo WDs in the field (Oppenheimer et al. 2001a) and in the Hubble Deep Field (Ibata et al. 2000; Méndez \& Minniti 2000) were later rejected by detailed model atmosphere analysis (see Bergeron et al. 2005, and references therein) and additional proper motion measurements (Kilic et al. 2004, 2005). To date, the coolest known probable halo WDs are WD 0346+246 (Hambly et al. 1997; Bergeron 2001) and SDSS J1102+4113, with $T_{\text {eff }} \approx 3800 \mathrm{~K}$ (Hall et al. 2008). There are also about a dozen ultracool WDs detected in the SDSS (Gates et al. 2004; Harris et al. 2008) that may be thick disk or halo WDs, but current WD atmosphere models have problems in reproducing their intriguing spectral energy distributions (SEDs). Therefore, their temperatures and ages remain uncertain.

Here, we report the identification of three old halo WD candidates discovered as part of our Bok and USNO proper motion survey. The details of this survey and our follow-up observations are discussed in Section 2, whereas our model fits and analysis are discussed in Section 3. 


\section{OBSERVATIONS}

In 2006 January, we started an $r$-band second-epoch astrometry survey with the Steward Observatory Bok 90 inch telescope with its 90Prime camera (Williams et al. 2001; Liebert et al. 2007). The 90Prime provides a field of view of $1.0 \mathrm{deg}^{2}$ with 0.45 pixel $^{-1}$ resolution. Since 2009 additional observations have been obtained with the U.S. Naval Observatory Flagstaff Station $1.3 \mathrm{~m}$ telescope using the CCD Mosaic Camera (1.4 $\mathrm{deg}^{2}$ field of view with $0^{\prime \prime} 6$ pixel $^{-1}$ resolution). We limited our program to the SDSS Data Release 3 footprint in order to have a relatively long time baseline between our program and the SDSS observations. We obtain proper motion errors of roughly $20 \mathrm{mas} \mathrm{yr}^{-1}$ at $r=21 \mathrm{mag}$ ( $g=22 \mathrm{mag}$ for cool WDs).

We select candidates for follow-up spectroscopy based on our proper motion measurements and the photometric colors. We further limit our sample to objects with high proper motion and relatively red colors in order to find the elusive thick disk and halo WDs. We started the follow-up optical spectroscopy of candidate halo WDs at the $6.5 \mathrm{~m}$ MMT equipped with the Blue Channel Spectrograph in 2009 June. Here, we present lowresolution spectroscopy of three halo WD candidates with $g-$ $i=1.5-1.75 \mathrm{mag}$. These observations were performed on UT 2009 June 19-21. Our targets are SDSS J213730.87+105041.6, $\mathrm{J} 214538.16+110626.6$, and $\mathrm{J} 214538.60+110619.0$ (hereafter $\mathrm{J} 2137+1050, \mathrm{~J} 2145+1106 \mathrm{~N}$, and $\mathrm{J} 2145+1106 \mathrm{~S}$, respectively). We used a 1 1.25 slit and the 500 line $\mathrm{mm}^{-1}$ grating in first order to obtain spectra with a wavelength coverage of 3660-6800 and a resolving power of $R=1200$. The $g$-band magnitudes of our targets range from 21.0 to $21.8 \mathrm{mag}$, and the exposure times range from 60 to 100 minutes. We obtained all spectra at the parallactic angle and acquired $\mathrm{He}-\mathrm{Ar}-\mathrm{Ne}$ comparison lamp exposures for wavelength calibration. We use the observations of the spectrophotometric standard star G24-9, which is also a cool WD, for flux calibration.

In addition, we obtained $J$ - and $H$-band imaging observations of our targets using the MMT and Magellan Infrared Spectrograph (MMIRS; McLeod et al. 2004) on the MMT on UT 2009 September 2 and 4. The FWHM of the images ranges from $0^{\prime} .8$ to $1^{\prime \prime} .3$. We use a $1^{\prime \prime} .0$ or $1^{\prime \prime} .4$ aperture for photometry. The $6.8 \times 6.8$ field of view of MMIRS enables us to use 20-50 nearby Two Micron All Sky Survey (2MASS) stars to calibrate the photometry. The optical and near-infrared photometry of our targets, as well as proper motions, are presented in Table 1 . The optical photometry is in the $\mathrm{AB}$ system and the $J H$ photometry is in the 2MASS (Vega) system. We use the corrections given in Eisenstein et al. (2006) to convert the SDSS photometry to the AB system. Two of our targets, J2145+1106N and S (N for north and $S$ for south), are separated by $10^{\prime \prime}$ and they have proper motions consistent within the errors. Hence, they are in a common proper motion binary system.

Out of the three targets, only $\mathrm{J} 2145+1106 \mathrm{~S}$ is detected in the USNO-B catalog, and it has a proper motion of $\mu_{\text {R.A. }}=181.3 \pm$ $5.2 \mathrm{mas} \mathrm{yr}^{-1}$ and $\mu_{\mathrm{decl}}=-367.7 \pm 5.2 \mathrm{mas} \mathrm{yr}^{-1}$ (Munn et al. 2004). These proper motion measurements are consistent with our measurements within the errors, and they demonstrate that our proper motion measurements are reliable.

\section{MODEL ATMOSPHERE ANALYSIS}

Our MMT spectroscopy shows that all three targets have featureless spectra; they are cool DC WDs. Below $5000 \mathrm{~K}$, $\mathrm{H} \alpha$ disappears in cool WD spectra. However, hydrogen can still show its presence through the red wing of Ly $\alpha$ absorption
Table 1

New Halo WD Candidates

\begin{tabular}{lccc}
\hline \hline \multicolumn{1}{c}{ Parameter } & $\mathrm{J} 2137+1050$ & $\mathrm{~J} 2145+1106 \mathrm{~N}$ & $\mathrm{~J} 2145+1106 \mathrm{~S}$ \\
\hline R.A. $^{\mathrm{a}}$ & $21: 37: 30.87$ & $21: 45: 38.16$ & $21: 45: 38.60$ \\
Decl. $^{\mathrm{a}}$ & $+10: 50: 41.6$ & $+11: 06: 26.6$ & $+11: 06: 19.0$ \\
$\mu_{\alpha}\left(\mathrm{mas} \mathrm{yr}^{-1}\right)$ & -228.9 & +191.9 & +185.9 \\
$\mu_{\delta}\left(\right.$ mas yr $\left.^{-1}\right)$ & -473.6 & -366.9 & -367.7 \\
$u$ & $23.31 \pm 0.69$ & $23.74 \pm 0.91$ & $23.45 \pm 0.75$ \\
$g$ & $21.77 \pm 0.06$ & $21.45 \pm 0.05$ & $21.00 \pm 0.03$ \\
$r$ & $20.51 \pm 0.03$ & $20.27 \pm 0.03$ & $19.93 \pm 0.02$ \\
$i$ & $20.02 \pm 0.03$ & $19.75 \pm 0.02$ & $19.49 \pm 0.02$ \\
$z$ & $19.73 \pm 0.08$ & $19.68 \pm 0.07$ & $19.38 \pm 0.06$ \\
$J$ & $19.21 \pm 0.10$ & $18.87 \pm 0.07$ & $18.54 \pm 0.06$ \\
$H$ & $19.25 \pm 0.18$ & $19.00 \pm 0.10$ & $18.31 \pm 0.06$ \\
$T_{\text {eff }}(\mathrm{K})$ & 3780 & 3730 & 4110 \\
Age $(\mathrm{Gyr})$ & 9.6 & 9.7 & 8.7 \\
Distance $^{\mathrm{b}}\left(\mathrm{pc}^{\mathrm{c}}\right)$ & 78 & 69 & 70 \\
$V_{\text {tan }}\left(\mathrm{km} \mathrm{s}^{-1}\right)$ & 195 & 136 & 136 \\
$U, V, W\left(\mathrm{~km} \mathrm{~s}^{-1}\right)$ & $172,-97,-35$ & $31,-75,-102$ & $31,-75,-102$
\end{tabular}

Notes.

a Coordinates are given for equinox J2000.0 at the observed epoch of 2001.7.

b These estimates are for $M=0.58 M_{\odot}(\log g=8.0)$. Ages, distances, and velocities depend strongly on the assumed mass (see Section 4.2).

(Kowalski \& Saumon 2006) in the blue and through collisioninduced absorption due to molecular hydrogen in the infrared (Hansen 1998; Saumon \& Jacobson 1999). Cool helium atmosphere WDs do not suffer from these opacities, and they are expected to show SEDs similar to blackbodies (Kowalski et al. 2007). Therefore, ultraviolet and near-infrared data are crucial for determining the atmospheric composition of cool WDs.

We use state-of-the-art WD model atmospheres to fit the optical and near-infrared photometry of our targets. The model atmospheres include the Ly $\alpha$ far red wing opacity (Kowalski \& Saumon 2006) as well as non-ideal physics of dense helium that includes refraction (Kowalski \& Saumon 2004), ionization equilibrium (Kowalski et al. 2007), and the non-ideal dissociation equilibrium of $\mathrm{H}_{2}$ (Kowalski 2006). Since parallax measurements are unavailable, we assume a surface gravity of $\log g=$ $8\left(M \approx 0.58 M_{\odot}\right)$. We discuss the implications of this mass assumption in Section 4.2.

We find that the observed SEDs of our targets are best matched by pure hydrogen atmosphere models. Figure 1 presents the observed and best-fit SEDs for our targets assuming a pure hydrogen atmosphere composition. The temperatures for these models range from 3730 to $4110 \mathrm{~K}$. The SEDs peak around $1 \mu \mathrm{m}$. Even though the optical portion of the SEDs may be explained by simple blackbodies, our $J$ - and $H$-band data show that they differ from blackbodies in the infrared. The pure hydrogen atmosphere models match the ultraviolet, optical, and near-infrared SEDs of our targets fairly well.

The fits for $\mathrm{J} 2137+1050$ and $\mathrm{J} 2145+1106 \mathrm{~N}$ are similar to the fits obtained for the halo WD candidate SDSS J1102+4113 (Hall et al. 2008). There are slight differences between the observations and these models. The synthetic $i$-band fluxes seem lower than that observed, and there are related problems with matching the $z$ - and $J$-band fluxes. Systematic problems most likely exist for models below $4000 \mathrm{~K}$. The models for the two coolest stars predict absorption bumps around $1 \mu \mathrm{m}$. These bumps have never been observed in the spectra of real WDs, indicating that the current collision-induced opacity calculations may be problematic for high-density atmospheres of cool WDs (see, e.g., Oppenheimer et al. 2001b; Bergeron \& Leggett 


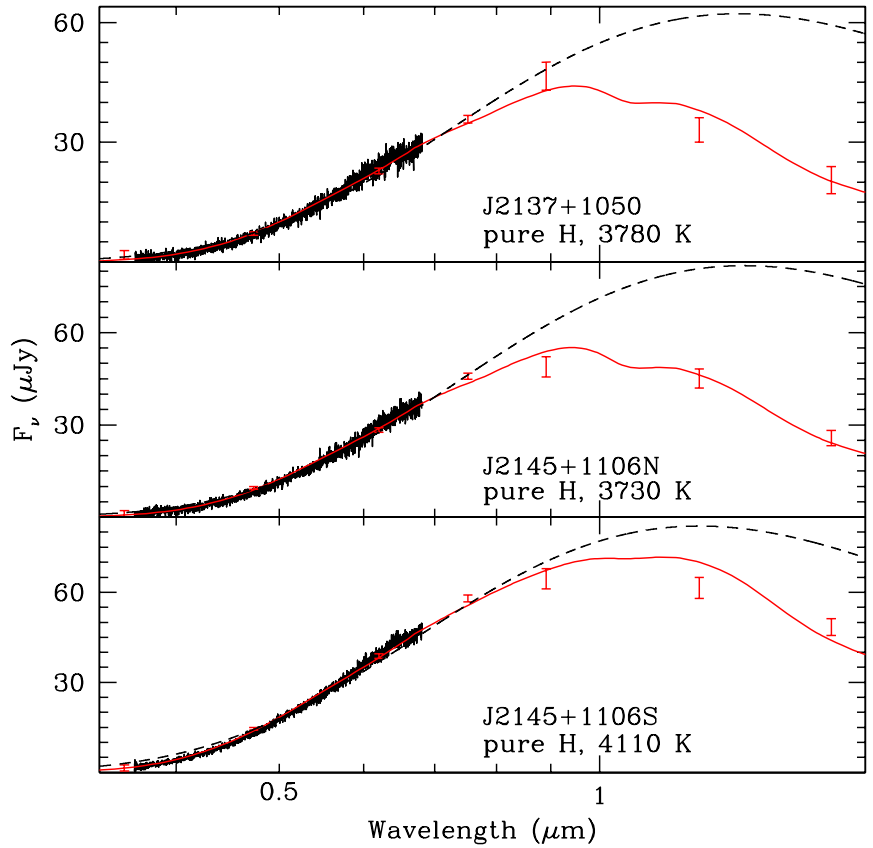

Figure 1. Observed spectra, SDSS photometry, and near-infrared photometry of our targets compared to the best-fit pure hydrogen atmosphere model spectra (solid lines, assuming $\log g=8$ ) and blackbody SEDs with the same temperatures (dashed lines).

(A color version of this figure is available in the online journal.)

2002; Kilic et al. 2009). Nevertheless, the overall SEDs of our targets agree with model predictions over the entire $0.3-1.7 \mu \mathrm{m}$ range.

Addition of helium can improve the model fits slightly. Figure 2 shows the best-fit models assuming pure $\mathrm{H}$, pure $\mathrm{He}$, and mixed $\mathrm{H} / \mathrm{He}$ composition. Since mixed $\mathrm{H} / \mathrm{He}$ atmospheres have higher pressure than pure $\mathrm{H}$ atmospheres at the same temperature, the collision-induced absorption is expected to be stronger. Addition of 4\%-16\% helium (relative to hydrogen) into the atmosphere helps in fitting the infrared portion of the SEDs. However, these fits are marginally better than the pure hydrogen atmosphere model fits, and they are not statistically significant. The best-fit temperature values are also similar to the pure hydrogen atmosphere solutions. Hence, the choice of a pure hydrogen or mixed $\mathrm{H} / \mathrm{He}$ composition with small amounts of helium does not significantly change our results. In any case, the good match between the optical spectrum and the models including Ly $\alpha$ absorption indicates that these WDs have hydrogen-dominated atmospheres; helium-dominated or highly helium-enriched atmospheres are ruled out (see also Hall et al. 2008).

The temperature, WD cooling age, and distance estimates for our targets based on pure hydrogen atmosphere models with $\log g=8\left(M=0.58 M_{\odot}\right)$, and the cooling models by Fontaine et al. (2001) are given in Table 1. This mass assumption implies that our targets are located at $70-80 \mathrm{pc}$ away from the Sun, and the WD cooling ages are $8.7 \mathrm{Gyr}$ or longer. Our model fits to the individual SEDs give cooling ages of 8.7-9.7 Gyr and distances of 69 and $70 \mathrm{pc}$ for the members of the J2145+1106 common proper motion system. The difference in cooling ages can be explained by a small mass difference between the two stars. These results suggest that the J2145+1106 system is a physical binary and that our model fits are reliable. Based on our proper motion measurements and assuming zero radial velocity, we also estimate the tangential velocity and Galactic

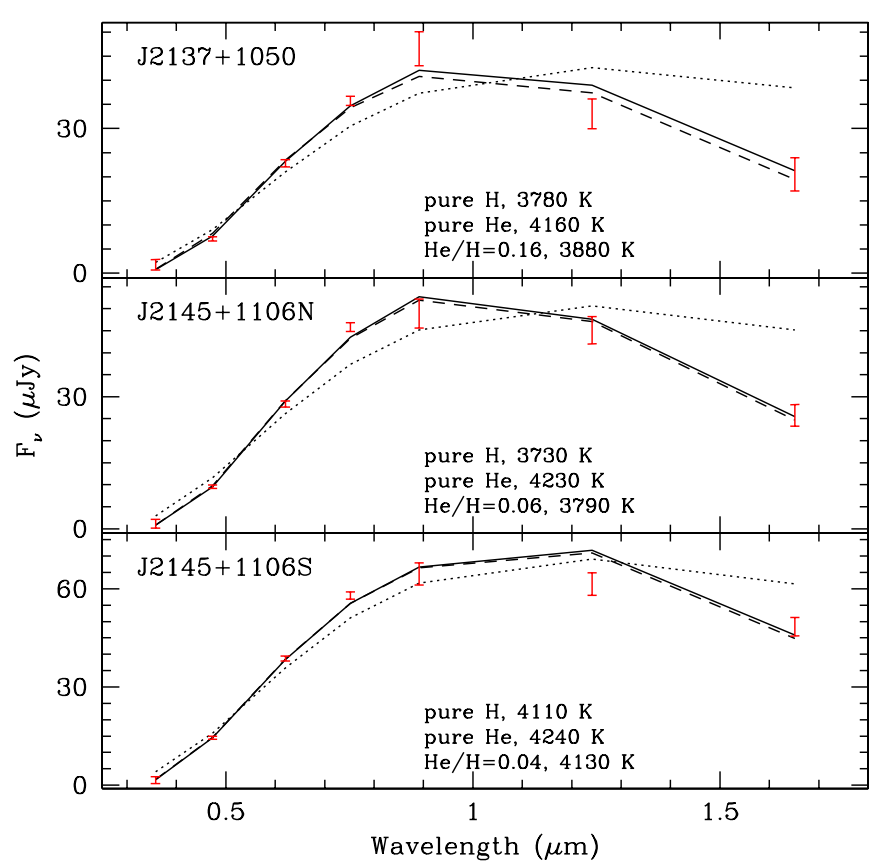

Figure 2. Comparison of the observed photometry (error bars) with the synthetic photometry from the best-fit pure hydrogen (solid lines), pure helium (dotted lines), and mixed $\mathrm{H} / \mathrm{He}$ (dashed lines) atmosphere models. The best-fit temperatures are given in each panel.

(A color version of this figure is available in the online journal.)

$U V W$ velocities for our targets. These WDs display tangential velocities of $140-200 \mathrm{~km} \mathrm{~s}^{-1}$.

\section{THICK DISK OR HALO?}

\subsection{Total Ages}

Bergeron et al. (2005) emphasize the importance of determining total stellar ages in order to associate any WD with the thick disk or halo. Modeling the optical and near-IR SEDs of the Oppenheimer et al. (2001a) WD sample, Bergeron et al. (2005) find that many of the WDs in that sample are fairly warm and too young to be halo WDs unless they all have masses near $0.5 M_{\odot}$. They find that, with estimated temperatures of $3950-4100 \mathrm{~K}$ and ages of 8.8-9.1 Gyr, F351-50 and WD 0351-564 are the two most likely halo candidates in the Oppenheimer et al. (2001a) sample.

For an average mass of $0.58 M_{\odot}$, our temperature estimates correspond to WD cooling ages of 9.6-9.7 Gyr for J2137+1050 and J2145+1106N. These two stars are the coolest field WDs currently known. Although the ultracool WDs discovered by Gates et al. (2004) and Harris et al. (2008) are possibly cooler than our targets, current models have problems in explaining the observed SEDs of these WDs (Bergeron \& Leggett 2002). Using the initial-final mass relations of Williams et al. (2009), Kalirai et al. (2008), and Salaris et al. (2009), we estimate that a $0.58 M_{\odot}$ WD would be the descendant of a 1.7-1.9 $M_{\odot}$ star. Such a progenitor halo star has a main-sequence lifetime of 1.0-1.3 Gyr (Marigo et al. 2008). Therefore, the total ages of our three targets range from 9.7 to $11.0 \mathrm{Gyr}$; they most likely belong to the halo or thick disk. The theoretical uncertainties due to the unknown core composition, helium layer mass, crystallization, and phase separation are on the order of 1-2 Gyr for these ages (Wood 1992; Montgomery et al. 1999; M. Montgomery 2010, private communication). 

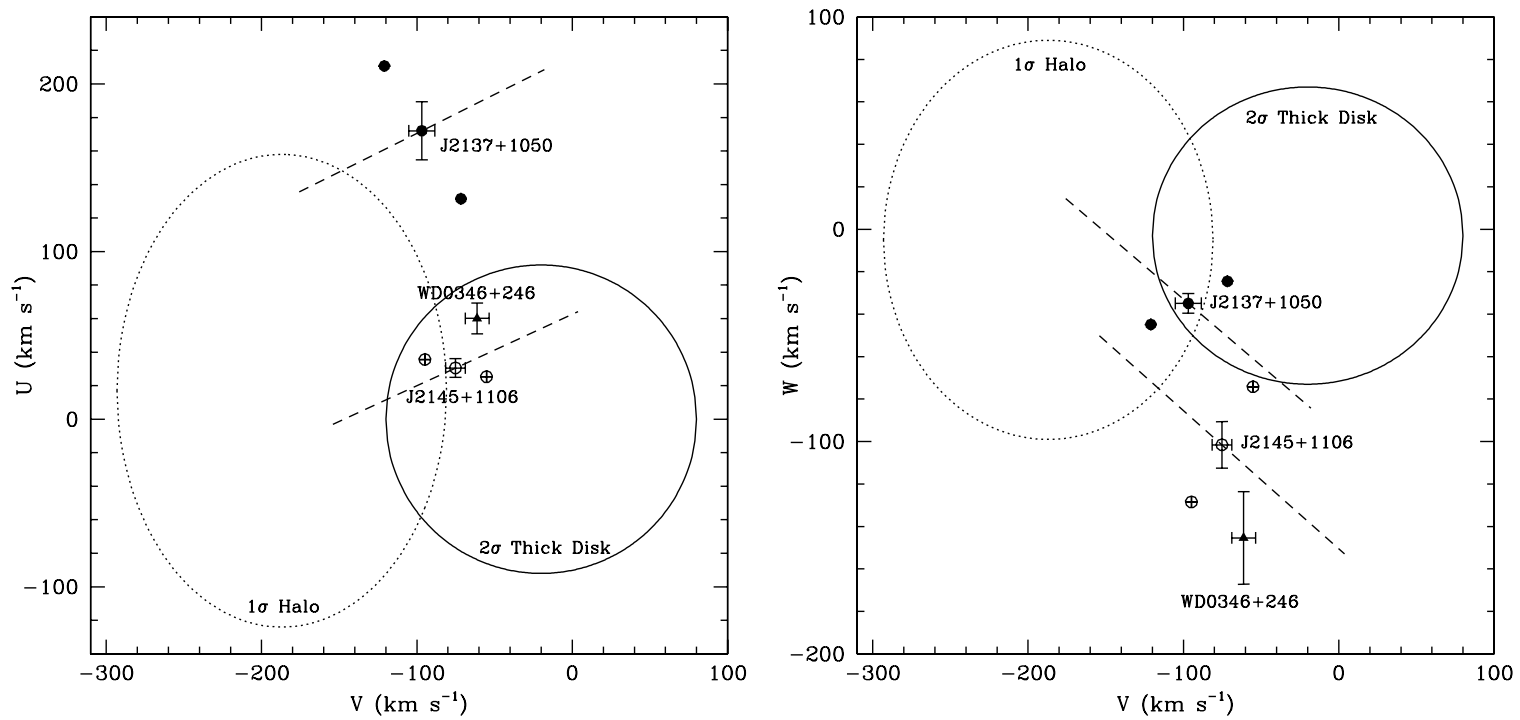

Figure 3. $U, V$, and $W$ space velocities for our targets assuming $0 \mathrm{~km} \mathrm{~s}^{-1}$ radial velocity and $\log g=7.5,8.0$, and 8.5 (from left to right). The points with error bars correspond to $\log g=8$. The probable halo member WD $0346+246$ is shown for comparison. The $2 \sigma$ velocity ellipse of the thick disk and the $1 \sigma$ ellipse of the halo are also shown. The dashed lines show the effect of changing the radial velocity from -100 to $+100 \mathrm{~km} \mathrm{~s}^{-1}$ (from left to right).

\subsection{Kinematic Membership}

Figure 3 shows the $U V W$ velocities of our targets, assuming that they have $0 \mathrm{~km} \mathrm{~s}^{-1}$ radial velocities, compared to the $1 \sigma$ velocity ellipse of the halo and $2 \sigma$ ellipse of the thick disk (Chiba \& Beers 2000). The velocities for the probable halo object WD $0346+246$ are also shown for comparison. The $U$ velocity of $\mathbf{J} 2137+1050$ is more than $3 \sigma$ different than the thick disk objects studied by Chiba \& Beers (2000). Similarly, the $W$ velocity of $\mathrm{J} 2145+1106$ is inconsistent with thick disk objects. The $U V W$ velocities of the $\mathrm{J} 2145+1106$ binary are similar to that of WD $0346+246$. The radial velocity assumption does not change these results. Negative radial velocities bring the $U V W$ velocities closer to the $1 \sigma$ distribution for the halo, and positive radial velocities move them away from the $2 \sigma$ thick disk distribution (see the dashed lines in Figure 3 ). Hence, both $\mathrm{J} 2137+1050$ and $\mathrm{J} 2145+1106$ systems most likely belong to the halo.

Without a parallax measurement, our age, distance, and velocity estimates are of course uncertain. A $\log g$ of $8.5(M=$ $0.9 M_{\odot}$ ) would imply WD cooling ages of 10.1-10.6 Gyr and $U V W$ velocities that are still inconsistent with the $2 \sigma$ thick disk velocity distribution. Likewise, a $\log g$ of $7.5\left(M=0.3 M_{\odot}\right)$ would imply WD cooling ages of 4.1-5.0 Gyr and $U V W$ velocities that are even more inconsistent with the thick disk sample (see Figure 3). The main-sequence lifetimes would be greater than the age of the universe unless the systems are unresolved double degenerates. An additional constraint is that $\mathrm{J} 2145+1106$ is a binary with a separation of $10^{\prime \prime}$ (700 AU, assuming $\log g=8$ ). This separation is too large to cause any effect on the evolution of each component, and it is small enough that the system can survive the gravitational perturbations from passing stars or Galactic tides for billions of years (Jiang \& Tremaine 2010 demonstrate that more than $99.9 \%$ of the binary stars with initial separations of $0.017 \mathrm{pc}(\approx 3500 \mathrm{AU})$ survive for a Hubble time). A scenario involving low-mass WDs would require both components of the $\mathrm{J} 2145+1106$ system to be double degenerates, which seems unlikely. In addition, our pure hydrogen atmosphere models with $\log g=8$ fit the SEDs better than the models with $\log g=7.5$, indicating that our targets are not likely to be low-mass WDs.

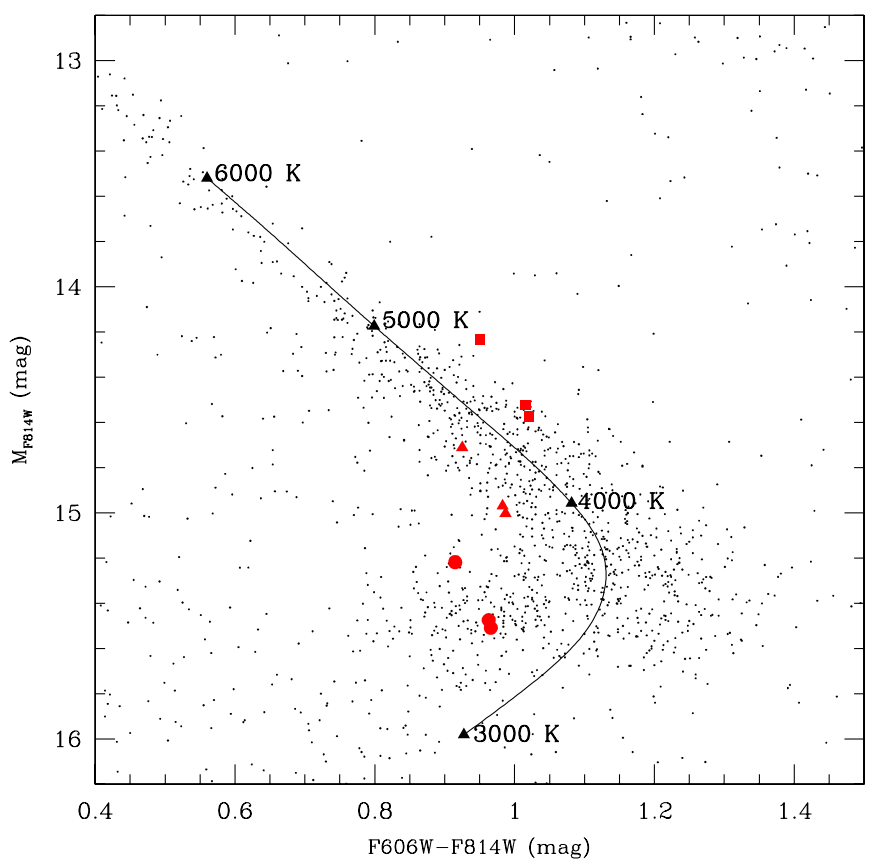

Figure 4. Color-magnitude diagram of the WDs in the globular cluster NGC 6397 (small dots; Hansen et al. 2007) and our three halo WD candidates assuming $\log g=7.5,8.0$, and 8.5 (from top to bottom). The NGC 6397 WD sequence is de-reddened by $E(F 606 W-F 814 W)=0.16$ and vertically shifted by $\mu=12.0$ mag (Kowalski 2007). The errors in reddening and distance modulus are on the order of 0.03 and $0.06 \mathrm{mag}$, respectively. The solid line shows the colors for $0.53 M_{\odot}$ WDs with $T_{\text {eff }}=3000-6000 \mathrm{~K}$.

(A color version of this figure is available in the online journal.)

Figure 4 displays a color-magnitude diagram of the point sources in the region that encloses the WD population of the globular cluster NGC 6397 and our three halo WD candidates assuming $\log g=7.5,8.0$, and 8.5. We use our best-fit WD model spectra to derive synthetic photometry in the $F 606 \mathrm{~W}$ and $F 814 W$ filters. Depending on the mass, our targets can fall on multiple parts of the WD cooling sequence of NGC 6397. If they are similar to the WDs in NGC 6397, they should have masses ranging from $0.5 M_{\odot}$ to $0.9 M_{\odot}(\log g=7.9-8.5)$. 
Kalirai et al. (2009) find the masses of the brightest WDs in the globular cluster M4 to be $0.53 M_{\odot}$. This is a reasonable lower limit for our targets assuming single star evolution. At $0.53 M_{\odot}$, our targets would have WD cooling ages of 8.0-9.1 Gyr, distances of 72-81 pc, and progenitor masses of 1.25-1.48 $M_{\odot}$ (Williams et al. 2009; Kalirai et al. 2008). The main-sequence lifetimes would be 1.7-2.8 Gyr for the progenitor halo stars (Marigo et al. 2008), and the total ages would be 9.7-11.9 Gyr. The Galactic space velocities would be inconsistent with the thick disk velocity distribution.

\section{CONCLUSIONS}

$\mathrm{J} 2137+1050$ and $\mathbf{J} 2145+1106$ are cool WDs with hydrogendominated atmospheres. Our effective temperature estimates of $3730-3780$ K make J2137+1050 and J2145+1106N the coolest WDs known in the solar neighborhood. Our best-fit models imply total ages of $\approx 10-11 \mathrm{Gyr}$, distances of $70-80 \mathrm{pc}$, and Galactic space velocities that are inconsistent with thick disk population within $2 \sigma$. We conclude that these targets most likely belong to the halo. However, trigonometric parallax observations are required in order to constrain the distances, masses, and ages of our targets accurately. Such observations are currently underway at the MDM 2.4 m telescope.

Like WD 0346+246 and SDSS J1102+4113 (Bergeron 2001; Hall et al. 2008), our three halo WD candidates have hydrogenrich atmospheres. The oldest WDs are likely to accrete from the interstellar medium within their $\sim 10$ Gyr lifetimes and end up as hydrogen-rich WDs even if they start with a pure helium atmosphere. However, the current sample of halo WD candidates is not large enough to conclude that most or all of the oldest WDs are hydrogen rich. Observations of larger samples of field WDs will be necessary to check whether all WDs turn into hydrogenrich atmosphere WDs or not (see the discussion in Kowalski \& Saumon 2006).

The three targets that we present here make up only a small fraction of the halo WD candidates in our proper motion survey. Follow-up observations of these targets will be necessary to confirm many more halo WD candidates that can be used to study the age and age dispersion of the Galactic thick disk and halo. Already we can see, however, that these halo (or possibly thick disk) WDs indicate a gap of 1-2 Gyr between the star formation in the halo and the star formation in the disk at the solar annulus. Our observations further demonstrate that deep, wide-field proper motion surveys ought to find many old halo WDs. Using the Liebert et al. (1988) WD luminosity function for the Galactic thin disk and a single burst 12 Gyr old population with $10 \%$ and $0.4 \%$ local normalizations for the thick disk and halo, we estimate that there are 3200 thick disk and 140 halo WDs per $1000 \mathrm{deg}^{2}$ (for a Galactic latitude of $45^{\circ}$ ) down to a limiting magnitude of $V=21.5 \mathrm{mag}$ (our survey limit). Pushing the limiting magnitude down to $V=24 \mathrm{mag}$ and assuming $50 \%$ sky coverage, we estimate that future surveys like the Pan-STARRS and Large Synoptic Survey Telescope (LSST) will image $\sim 1.3$ million thick disk and $\sim 80,000$ halo WDs. These surveys will be invaluable resources for halo WD studies.

Support for this work was provided by NASA through the Spitzer Space Telescope Fellowship Program, under an award from Caltech. This material is also based on work supported by the National Science Foundation under grants AST-0607480 and AST-0602288. We thank the MMIRS commissioning team for obtaining the near-infrared observations, and J. Liebert for extensive help with the Bok telescope imaging observations and for many useful discussions. We also thank E. Olszewski for building the 90Prime instrument and the Steward Observatory Time Allocation Committee for supporting our proper motion survey program.

Facilities: MMT (Blue Channel Spectrograph and MMIRS)

\section{REFERENCES}

Bedin, L. R., Salaris, M., Piotto, G., Anderson, J., King, I. R., \& Cassisi, S. 2009, ApJ, 697, 965

Bergeron, P. 2001, ApJ, 558, 369

Bergeron, P., \& Leggett, S. K. 2002, ApJ, 580, 1070

Bergeron, P., Ruiz, M. T., Hamuy, M., Leggett, S. K., Currie, M. J., Lajoie, C.-P., \& Dufour, P. 2005, ApJ, 625, 838

Chiba, M., \& Beers, T. C. 2000, AJ, 119, 2843

Eisenstein, D. J., et al. 2006, ApJS, 167, 40

Fontaine, G., Brassard, P., \& Bergeron, P. 2001, PASP, 113, 409

Gates, E., et al. 2004, ApJ, 612, 129

Hall, P. B., Kowalski, P. M., Harris, H. C., Awal, A., Leggett, S. K., Kilic, M., Anderson, S. F., \& Gates, E. 2008, AJ, 136, 76

Hambly, N. C., Smartt, S. J., \& Hodgkin, S. T. 1997, ApJ, 489, L157

Hansen, B. M. S. 1998, Nature, 394, 860

Hansen, B. M. S., et al. 2002, ApJ, 574, L155

Hansen, B. M. S., et al. 2004, ApJS, 155, 551

Hansen, B. M. S., et al. 2007, ApJ, 671, 380

Harris, H. C., et al. 2006, AJ, 131, 571

Harris, H. C., et al. 2008, ApJ, 679, 697

Ibata, R., Irwin, M., Bienaymé, O., Scholz, R., \& Guibert, J. 2000, ApJ, 532, L41

Jiang, Y.-F., \& Tremaine, S. 2010, MNRAS, 401, 977

Kalirai, J. S., Hansen, B. M. S., Kelson, D. D., Reitzel, D. B., Rich, R. M., \& Richer, H. B. 2008, ApJ, 676, 594

Kalirai, J. S., Saul Davis, D., Richer, H. B., Bergeron, P., Catelan, M., Hansen, B. M. S., \& Rich, R. M. 2009, ApJ, 705, 408

Kilic, M., Kowalski, P. M., Reach, W. T., \& von Hippel, T. 2009, ApJ, 696, 2094 Kilic, M., Méndez, R. A., von Hippel, T., \& Winget, D. E. 2005, ApJ, 633, 1126 Kilic, M., von Hippel, T., Méndez, R. A., \& Winget, D. E. 2004, ApJ, 609, 766 Kilic, M., et al. 2006, AJ, 131, 582

Kowalski, P. M. 2006, ApJ, 641, 488

Kowalski, P. M. 2007, A\&A, 474, 491

Kowalski, P. M., \& Saumon, D. 2004, ApJ, 607, 970

Kowalski, P. M., \& Saumon, D. 2006, ApJ, 651, L137

Kowalski, P., et al. 2007, Phys. Rev. B, 2007, 76

Leggett, S. K., Ruiz, M. T., \& Bergeron, P. 1998, ApJ, 497, 294

Liebert, J., Dahn, C. C., \& Monet, D. G. 1988, ApJ, 332, 891

Liebert, J., Kilic, M., Williams, K. A., von Hippel, T., Winget, D. M. J., Harris, H. C., Levine, S., \& Metcalfe, T. S. 2007, in ASP Conf. Ser. 372, 15th European Workshop on White Dwarfs, ed. R. Napiwotzki \& M. R. Burleigh (San Francisco, CA: ASP), 129

Marigo, P., Girardi, L., Bressan, A., Groenewegen, M. A. T., Silva, L., \& Granato, G. L. 2008, A\&A, 482, 883

McLeod, B. A., Fabricant, D., Geary, J., Martini, P., Nystrom, G., Elston, R., Eikenberry, S. S., \& Epps, H. 2004, Proc. SPIE, 5492, 1306

Méndez, R. A., \& Minniti, D. 2000, ApJ, 529, 911

Montgomery, M. H., Klumpe, E. W., Winget, D. E., \& Wood, M. A. 1999, ApJ, 525,482

Munn, J. A., et al. 2004, AJ, 127, 3034

Oppenheimer, B. R., Hambly, N. C., Digby, A. P., Hodgkin, S. T., \& Saumon, D. 2001a, Science, 292, 698

Oppenheimer, B. R., et al. 2001b, ApJ, 550, 448

Salaris, M., Serenelli, A., Weiss, A., \& Miller Bertolami, M. 2009, ApJ, 692, 1013

Saumon, D., \& Jacobson, S. B. 1999, ApJ, 511, 107

Williams, G. G., Olszewski, E., Lesser, M., \& Burge, J. Steward Observatory 90-Prime Team 2001, BAAS, 33, 791

Williams, K. A., Bolte, M., \& Koester, D. 2009, ApJ, 693, 355

Winget, D. E., Hansen, C. J., Liebert, J., Van Horn, H. M., Fontaine, G., Nather, R. E., Kepler, S. O., \& Lamb, D. Q. 1987, ApJ, 315, L77

Wood, M. A. 1992, ApJ, 386, 539 